\title{
Optimization of microfluidic single cell trapping for long-term on-chip culture $\dagger$
}

\author{
Stefan Kobel, ${ }^{a}$ Ana Valero, ${ }^{b}$ Jonas Latt, ${ }^{c}$ Philippe Renaud ${ }^{b}$ and Matthias Lutolf ${ }^{\star a}$ \\ Received 1st September 2009, Accepted 3rd December 2009 \\ First published as an Advance Article on the web 13th January 2010 \\ DOI: 10.1039/b918055a
}

The poor efficiency of microfluidic single cell trapping is currently restricting the full potential of stateof-the-art single cell analyses. Using fluid dynamics simulations in combination with particle image velocimetry to systematically optimize trap architectures, we present a microfluidic chip with enhanced single cell trapping and on-chip culture performance. Upon optimization of trap geometries, we measured trapping efficiencies of up to $97 \%$. Our device also enables the stable, relatively long-term culture of individual non-adherent mammalian cells in high-throughput without a significant decrease in cell viability. As a first application of this platform we demonstrate the automated separation of the two daughter cells generated upon single cell division. The reliable trapping and re-trapping of mammalian cells should for example provide the fundament for novel types of investigations in stem cell and tumour cell biology, which depend on reliable tracking of genealogical relationships such as in stem cell lineage tracking.

\section{Introduction}

Single cell analysis holds much promise for applications in the Life Sciences. For example, genetic analyses of heterogeneous cell populations, ${ }^{1}$ hybridoma screenings, ${ }^{2}$ or fundamental biological studies on rare stem cells or tumor cells ${ }^{2}$ greatly benefit from reliable single cell handling techniques. ${ }^{3-5}$

One of the most robust methods to analyze populations of single cells in relatively high throughput are microwell arrays. ${ }^{6-9}$ These platforms allow thousands of single cells to be randomly captured by gravity at the bottom of a microwell and analyzed in cell culture. Microwells can be readily used to trap individual cells, given that cell adhesion is restricted to the bottom of the microwell such as those formed from a non-cell-adhesive material like poly(ethylene glycol) (PEG) hydrogels. ${ }^{7,8}$ However, microwell cultures are static, limiting their possibilities to actively manipulate the trapped cells, for example to conduct medium changes or to temporally control the exposure of the cells to mitogenic stimuli. On the other hand, microfluidic valves, optical tweezers, dielectrophoresis (DEP) and acoustic waves, typically integrated with microfluidic devices, ${ }^{10-12}$ provide powerful

${ }^{a}$ Laboratory of Stem Cell Bioengineering and Institute of Bioengineering, Ecole Polytechnique Fédérale de Lausanne (EPFL), CH-1015 Lausanne, Switzerland. E-mail: matthias.lutolf@epfl.ch; Fax: +41 (0) 2169396 65; Tel: +41 (0) 21693

${ }^{b}$ Laboratory of Microsystems, Ecole Polytechnique Fédérale de Lausanne (EPFL), CH-1015 Lausanne, Switzerland

${ }^{c}$ Laboratoire d'ingénierie numérique, Ecole Polytechnique Fédérale de Lausanne (EPFL), CH-1015 Lausanne, Switzerland

$\uparrow$ Electronic supplementary information (ESI) available: ESI Movie 1: Images of green fluorescent $500-\mathrm{nm}$ beads in the single cell trap taken at a 70-ms interval; ESI Movie 2 and 3: The trapping of single cells in a 1-mm and 4-mm device, respectively; ESI Fig 1: The simulated wall shear stresses in the main channel of a 2-mm design for different flow rates; ESI Fig 2: Survival of single cells statically cultured in micro-well arrays; ESI Table 1: Predicted shear stresses for the different flow rates used for on-chip single cell culture. See DOI: 10.1039/b918055a approaches to trap and manipulate single cells. ${ }^{13-17}$ However, optical tweezers or valve-controlled devices typically are able to handle only one or a few single cells at once, ${ }^{13,18,19}$ whereas DEPbased systems have a higher throughput, but are less suitable for long-term cell culture due to potentially cytotoxic low-conductivity buffers ${ }^{15}$ and/or high temperatures induced by Jouleheating. ${ }^{20}$

A family of emerging technologies that combines active single cell handling with the potential for high-throughput experimentation are microfluidic hydrodynamic traps. In these traps cells can be immobilized in narrow gaps, too small for cells to pass through. ${ }^{21-23}$ Cells are drawn into the trap by flow that can either be generated using pressure, ${ }^{24,25}$ pump driven control channels $^{22,26}$ or by re-connecting the gap to the main channel. ${ }^{21,23,27}$ In the latter case, one fraction of the flow crosses the trap and the other fraction passes through the main channel. ${ }^{28}$ Once the trap is filled, the flow across the trap is reduced and thus the number of trapped cells should be limited to one. ${ }^{23}$ A striking example of such a 'self-regulating' trap device has been presented by Tan and Takeuchi. ${ }^{29}$ Focusing on wellcontrolled polymer beads, these authors realized that the efficiency of hydrodynamic single bead trapping depends on the ratio of the fluxes through the trap and the main channel. Furthermore, they correlated this ratio with the fluidic resistance of the trap and the main channel and obtained a perfect bead trapping efficiency of $100 \%$ with a ratio of $1: 3 .{ }^{29}$ Whether this system would be applicable to single cell trapping was not investigated.

With very few exceptions, including the aforementioned work, ${ }^{29}$ single cell traps have so far been designed by intuition and optimized by trial-and-error approaches. As a consequence, single cell traps thus far are rather inefficient, with trapping yields of only 10 to $20 \%{ }^{27,30}$ Moreover, due to high shear stresses, ${ }^{27}$ conventional single cell traps do often impair cell survival and offer a relatively poor position accuracy during long-term experiments. ${ }^{30}$ Due to the high throughput of many trap arrays, 
such complexities may not be a problem in some cell assays, but it may be an issue when working with rare cells such as stem cells or hybridoma cells, where clonality and knowledge of the fate of a single cell is key.

To address these limitations, we combined fluid dynamics simulation with experimental approaches to optimize the efficiency of single cell trapping in traps of variable geometry. We systematically adapted the Tan and Takeuki microfluidic trap design optimized for beads ${ }^{29}$ for the capture of single mammalian cells. Indeed, our trap modifications resulted in stable single cell trapping with efficiencies of nearly $100 \%$. Flow rates were identified that tolerated a long-term cell survival of $95 \%$. We believe that this increased trapping efficiency affords cell assays that were previously impossible. As an example, we successfully demonstrated the 'automated' separation of daughter cells upon cell division using a series of perfused single cell traps. This is currently only possible using rather elaborate manual micromanipulation techniques.

\section{Materials and methods}

\section{Microfabrication of the microfluidics chip}

The microfluidic chips were molded in PDMS from a two-layered SU8 mold as previously described ${ }^{23,31}$ and irreversibly bonded onto a glass slide. Briefly, a $3 \mu \mathrm{m}$ thick layer of SU8 GM1040 (Gersteltec, Switzerland) was spin coated onto a silicon wafer containing etched alignment marks. The wafer was then aligned and exposed to the first mask containing the trap connection using a Suess MA6. After the post exposure bake, the second layer of SU8 (GM1060) was spin coated to a thickness of $15 \mu \mathrm{m}$ and exposed to the second mask containing only the main channels. The wafer was post-exposure baked, developed with IPA (isopropyl alcohol) and diced with a dicing machine (Disco DAD321). The thickness of the two SU8 layers was confirmed with a surface profiler (Alpha-Step 500, Tencor).

The microstructured wafer was then used to mold PDMS (Sylgard $^{\circledR} 184$ Silicone Elastomer, Dow Corning Corporation, USA). The components were mixed in a weight ratio of 10 parts of base to one part of curing agent, degassed under vacuum and injected into a custom-made mold containing the wafer and up to eight metal pins to form the inlets. ${ }^{32}$ After curing the PDMS in an oven for $12 \mathrm{~h}$ at $65{ }^{\circ} \mathrm{C}$, the mold was disassembled and the resulting PDMS chips were bonded onto a glass slide after a brief oxygen plasma treatment.

\section{Fluid dynamics simulation using lattice Boltzmann}

In order to study the hydrodynamic conditions, a fluid flow through a single cell trap was simulated using the open source lattice Boltzmann library Palabos version 0.6 (available at www.lbmethod.org/palabos). Three models were programmed based on the dimensions measured from confocal images with main channel lengths of 1,2 or $4 \mathrm{~mm}$, respectively. The simulation used a Bhatnagar-Gross-Krook (BGK) model with a D3Q19 lattice ${ }^{33}$ on a homogeneous, regular grid with a resolution of one grid node for a physical length of $1 \mu \mathrm{m}$. The no-slip walls of the channels were modeled through a bounce-back scheme, while a constant velocity was imposed on the inlet, and a fixed pressure on the outlet, by means of a regularized boundary condition. ${ }^{34}$ The simulations were executed in parallel on 100 cores of a commodity cluster with Intel Xeon E5440 quad-core processors at $2.8 \mathrm{GHz}$ with a Gigabit Ethernet interconnection network. To simulate the three different design variants, the Mach number and the Reynolds number were set to $\mathrm{Ma}=10^{-3} \sqrt{3}$ and to $\mathrm{Re}=0.01958$, respectively, corresponding to a flow rate of $1 \mu \mathrm{l} / \mathrm{min}$ in the entire array of 64 parallel traps. To predict the stress at flow rates of 20,100 and $500 \mathrm{nl} / \mathrm{min}$ the numerical data was extrapolated from simulations of the 2-mm design at flow rates of $1 \mu \mathrm{l} / \mathrm{min}$ to $1000 \mu \mathrm{l} / \mathrm{min}(\mathrm{Re}=19.58)$. Velocities, density and shear stress were regularly saved as VTK files and visualized using the open source program Paraview (available at www.paraview.org).

\section{Determination of the flow profile using particle image velocimetry}

To assess the efficiency of the single cell trap, microfluidic chips with arrays of traps with a main channel length of 1,2 or $4 \mathrm{~mm}$, respectively (64 traps in parallel and 3 to 9 traps in series, depending on main channel length) were bonded to a cover slide. The chip was then connected to a high-precision syringe pump (neMESYS, Cetoni GmbH, Germany) equipped with a $100 \mu \mathrm{l}$ syringe (ILS, Germany) that was loaded with green fluorescent $0.5 \mu \mathrm{m}$ beads in water with $0.1 \%$ Triton-X100 (Sigma, Switzerland). After priming the chip at a flow rate of 5 to $10 \mu \mathrm{l} / \mathrm{min}$, the chip was perfused at a flow rate of $0.1 \mu \mathrm{l} / \mathrm{min}$ and allowed to equilibrate for 5 to $10 \mathrm{~min}$. Sequential images of the moving beads were acquired at a time interval of $70 \mathrm{~ms}$ and a scanning frequency of $8 \mathrm{MHz}$ using a confocal microscope SP5 (Leica, Germany) equipped with a resonance scanner and an immersion objective with a magnification of $100 \times$ and a numerical aperture of $1.45 .^{35}$ The scanning area and focal plane were positioned to simultaneously image the middle of the main channel and the exit channel of a trap.

Flow velocities were obtained by automatically tracking the beads using the object tracker application of MetaMorph, a programmable image acquisition and analysis software (Molecular Devices, California, USA). In brief, this plug-in semiautomatically identifies and tracks objects by detecting the displacement of intensity profiles over several frames (see for detailshttp://www.moleculardevices.com/pages/software/metamorph.html). Because the plug-in allows the definition of the expected migration direction, wrong correlations of neighboring beads can be minimized. The velocities of several hundreds of beads were then plotted against their position in the microchannel cross-section to obtain the velocity profile. The ratios of the fluxes through the main channel and the trap were determined as the ratio of the maximum velocities in the looped main channel and in the trap, which was defined as the difference of the maximum velocity of the main channel and the exit channel. ${ }^{36}$

\section{Cell culture}

EG7 cells, a non-adherent T-cell lymphoma cell line, were cultured in an incubator $\left(5 \% \mathrm{CO}_{2}, 37^{\circ} \mathrm{C}\right)$ as a suspension culture in RMPI 1640 medium containing Glutamax (Invitrogen, Switzerland), supplemented with $10 \%$ fetal bovine serum (FBS), $10 \mathrm{nM}$ HEPES, $100 \mathrm{U} / \mathrm{ml}$ Penicillin-Streptomycin, $50 \mu \mathrm{M}$ 
$\beta$-mercaptoethanol (all from Invitrogen, Switzerland) and $400 \mu \mathrm{g} / \mathrm{ml} \mathrm{G} 418$-sulfate solution (Brunschwig, Switzerland). For confocal microscopy, cells were labeled using a membrane staining kit (PKH26, Sigma, Switzerland) according to the manufacturer's instructions.

\section{Determination of the trapping efficiency}

To assess the efficiency of single cell trapping, microfluidic chips with arrays of traps with a main channel length of 1,2 or $4 \mathrm{~mm}$, respectively (64 traps in parallel and 3 to 10 traps in series, depending on main channel length) were designed and fabricated as described above. Each chip consisted of two inlets, one for the medium perfusion and one to for cell loading. The chip was first primed with complete cell culture medium at a flow rate of $25 \mu \mathrm{l} / \mathrm{min}$ and cells were injected through the spare inlet. After closing the cell inlet with a plug, the chip was placed on an inverted microscope (Axio Observer Z1, Zeiss), perfused at a flow rate of $20 \mathrm{nl} / \mathrm{min}$ and the ratio of successful and failed trapping events was quantified. In order to analyze a sufficiently large number of trapping events for statistical analysis, already trapped cells were removed from the traps by reversing the flow direction for approximately 1.5 to $2 \mathrm{~s}$ and restarting the perfusion. Each chip design was tested in triplicate with over 50 repetitions per experiment.

\section{Time-lapse microscopy to assess long-term trapping stability and cell viability}

In order to determine cell viability and long-term trapping stability, time-lapse experiments were performed on an automated cell imager (BD Pathway 855) with environmental chamber $\left(37^{\circ} \mathrm{C}, 5 \% \mathrm{CO}_{2}\right)$. Chips with 2 or $4 \mathrm{~mm}$ main channel lengths and tubings (1/32" ID teflon tubings) were first washed with a $1 \%$ Triton $\mathrm{X} 100$ in $\mathrm{ddH}_{2} \mathrm{O}$ (Sigma-Aldrich) solution, sterilized with $70 \% \mathrm{EtOH}$, and then primed with complete cell culture medium containing $2 \mu \mathrm{g} / \mathrm{ml}$ propidium iodine (PI) to detect cell death. After cell loading, chips were perfused at 20 , 100 and $500 \mu \mathrm{l} / \mathrm{min}$ and images acquired every $30 \mathrm{~min}$ for $12 \mathrm{~h}$. For comparison, single cells were seeded onto a hydrogel microwell array and analyzed using identical conditions. ${ }^{8}$

\section{Results and discussion}

\section{Design of an efficient microfluidic single cell trap array}

Due to the simplicity and elegance of valve-free bead trapping, we chose to adapt a previously developed microfluidic single bead trap for the capture of single mammalian cells. ${ }^{29}$ Our design principally followed the structure published by Tan and Takeuchi and consisted of a square-wave shaped main channel and a cavity to host a single cell (Fig. 1A). ${ }^{29}$ However, instead of working with a vertical gap, that is, a very narrow microchannel with the same height as the main channel, we altered the trap structure to consist of a $3-\mu \mathrm{m}$ high and $12-\mu \mathrm{m}$ wide horizontal gap (Fig. 1B). We hypothesized that this design variant would allow a more robust microfabrication and a better control over the number of cells per trap. ${ }^{23}$

To study single cell trapping with different flow profiles, we designed three devices modulating the predicted ratio of the
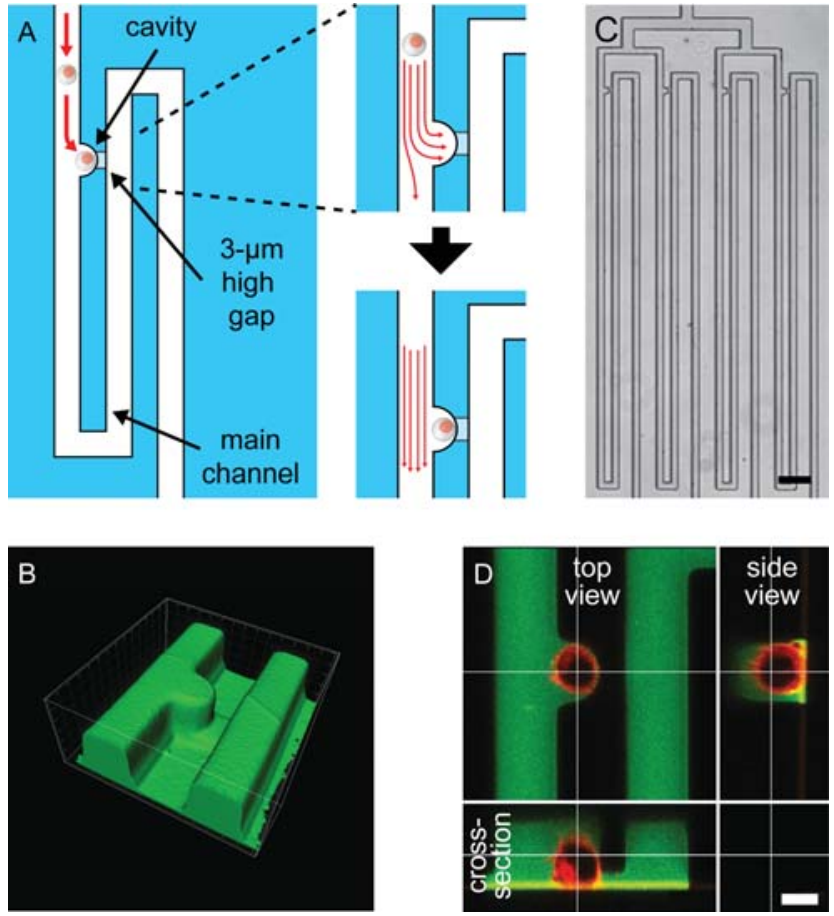

Fig. 1 Principle of single cell trapping. (A) Schematic illustration of the single cell trap with two inserts describing the fluid flow in the trap. The trap consists of a long looped main channel, a cavity for a single cell and a $3-\mu \mathrm{m}$ high gap (the device is not drawn to scale). Media flow and cells enter the trap from the top, where the majority of the flow will pass through the trap due to high resistance of the long main channel, and drag the cell into the trap. Once trapped, the cell will be maintained stably in the trap due to the pressure difference between the inlet and the outlet and the flow is re-directed to the main channel. (B) A three-dimensional (3D) reconstruction of the trap imaged by confocal laser scanning microscopy. (C) A section of an array of 64 (parallel) $\times 9$ (in series) single cell traps of $1 \mathrm{~mm}$ main channel length (scale bar is $100 \mu \mathrm{m}$ ). (D) An orthogonal view of a fluorescently labeled, trapped cell demonstrating that the cavity is too small for the cell to pass through (scale bar is $10 \mu \mathrm{m}$ ).

fluxes through the trap and the main channel from $1: 1,1: 2$ and $1: 4$, respectively. Using the model presented by Tan and Takeuchi, ${ }^{29,37}$ we varied the length of the main channel size from 1 to $4 \mathrm{~mm}$. Each chip consisted of 64 parallel channels with 9 to 3 traps in series (depending on the main channel length) yielding trap densities from 700 to 175 traps $/ \mathrm{cm}^{2}$ (Fig. 1C).

We chose to use a two-layered SU8 mold for the soft lithography process $^{23}$ because it enabled a more precise microfabrication of the 3- $\mu \mathrm{m}$ high gap than is the case for a narrow high-aspect ratio gap. ${ }^{31}$ This also allowed us to exactly match the dimensions of the gap to the diameter of a single cell (Fig. 1D). Indeed, we never observed multiple cells in one trap, a common problem associated with other single cell trap designs, ${ }^{23,27,28}$ because the trapped cell efficiently blocked the gap on its entire cross-section, thus eliminating any flow that could potentially trap additional cells (Fig. 1A).

In addition, horizontal gaps require the microchannel height to be close to the diameter of a single cell to prevent stacking of several cells in a trap (data not shown), ${ }^{27,29}$ thereby risking that the microchannels are blocked by stacked cells at low flow rates. 
Using a two-layered mold, we could choose the dimensions of the main channel to be about $30 \%$ to $40 \%$ larger than the average diameter of a single cell and minimize this risk.

\section{Computational simulations to visualize and predict flow profiles of microfluidic single cell traps}

To study the mechanisms of microfluidic cell trapping in a controllable environment, the flow profile in a single cell trap was simulated numerically using a lattice Boltzmann method ${ }^{33,38}$ (see also www.lbmethod.org), a method based on a discretization of the Boltzmann equation for the statistics of a gas. To guarantee an optimal numerical accuracy, all length scales were fully resolved, using a direct numerical simulation and a homogeneous, regular grid. Because the implementation of large models in a massively parallel environment is simple with lattice Boltzmann, independently of the complexity of the simulated domain, we were able to simulate our trap design over a wide range of main channel lengths within a few hours (Fig. 2A).

Because an efficient single cell trap should be designed to increase the probability of the center of mass of a cell moving within the streamlines passing through the trap, one rationale for these simulations was to determine the shape and the origin of the flow fraction crossing the trap. A plot of the streamlines starting at the gap helped to identify the convexly contoured profile of this flow and to show that the other flow fraction passing through the main channel originates from the corner most distant to the gap (Fig. 2B).

In the simulations, the Reynolds number was chosen as $\mathrm{Re}=0.02$, corresponding to a flow rate of $1 \mu \mathrm{l} / \mathrm{min}$ for an entire array of 64 parallel traps, which is higher than the Reynolds numbers used in the microfluidic experiments. Because the computational costs increase at a rate inversely proportional to the Reynolds number, it was not convenient to further lower this parameter in the simulation. However, the discrepancy between experiment and simulation can be considered irrelevant, because in both cases the flow rates are situated in a so-called creeping flow regime. In this regime, the Reynolds number is sufficiently small to allow the non-linear term of the Navier-Stokes equations to be neglected, and the solution of the equations then depends linearly on the Reynolds number. ${ }^{39}$

Under these assumptions, we determined the wall shear stress at different flow rates (ESI Fig. $1 \mathrm{~B} \dagger$ ) and used these data to extrapolate the shear stress for flow rates used in other studies, ${ }^{23}$ showing that we can reach shear stresses in the order of a few $\mathrm{mPa}$ (ESI Table $1 \dagger$ ). Such shear stresses can be found in tissues due to interstitial flow $^{40}$ and are also comparable to the shear stress found in other microfluidic devices. ${ }^{23,30}$
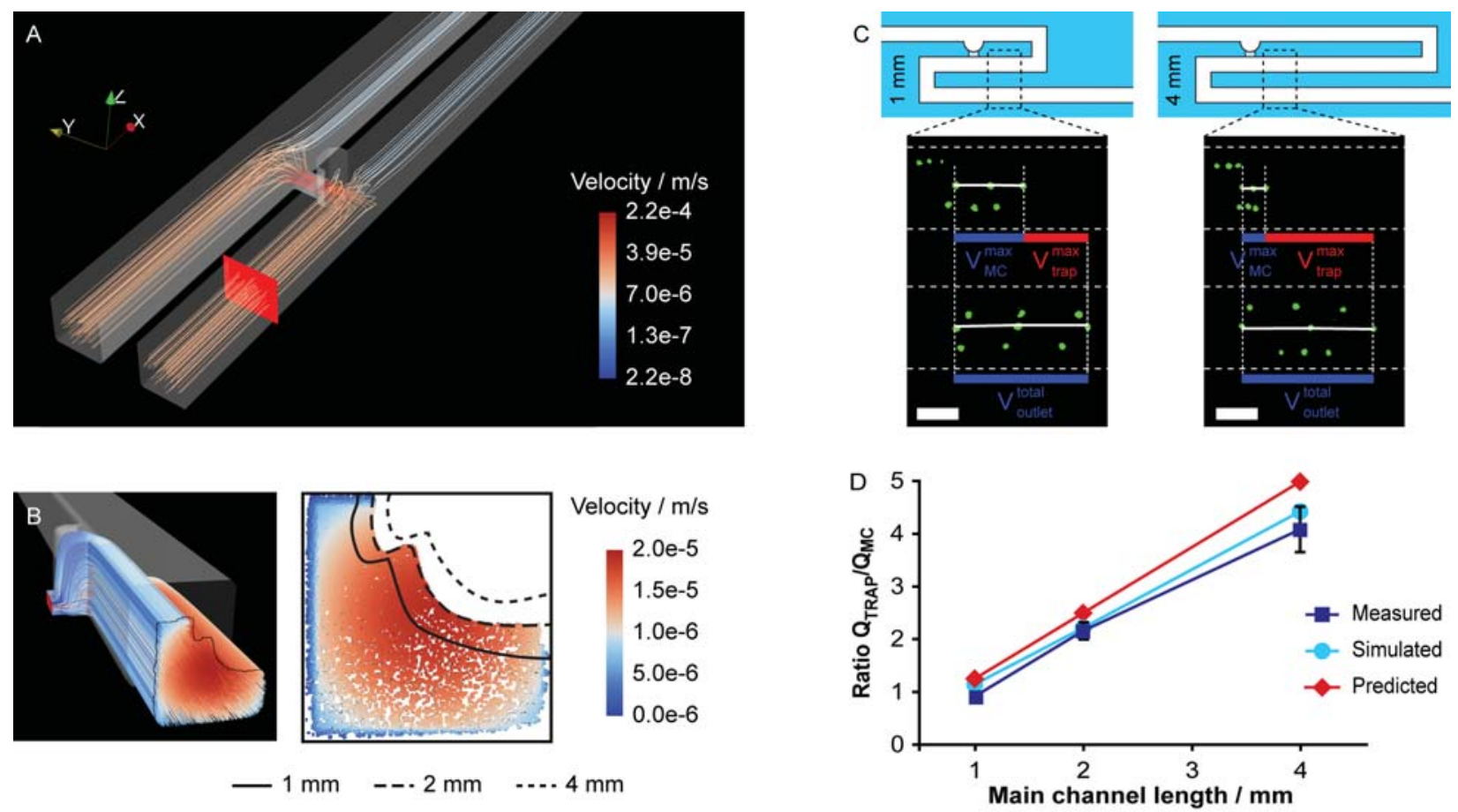

Fig. 2 Characterization of the hydrodynamic profile of the single cell trap using computational modeling and particle image velocimetry (PIV). (A) A streamline plot in the central part of the single cell trap (2-mm design) illustrating the two flow fractions passing through the trap and the main channel (MC). The colors of the streamlines represent their velocity on a logarithmic scale. (B) Three-dimensional (3D) plot and cross-section through the inlet (red section in (A)) of the streamlines that originate inside the trap, illustrating the convexly shaped fraction of the streamlines that pass through the $3 \mu \mathrm{m}$ gap. A cell with its centre of mass moving along these streamlines will be dragged into the trap. The surfaces of the corresponding cross-sections of the 1-mm and 4-mm designs are indicated as well and show the increase of the size of the flow fraction passing the trap. The total surface area occupied by the flow fraction passing through the trap increases from $63 \%$ to approximately $80 \%$. (C) Overlay of images of 500-nm beads in the main channel and the outlet of the single cell trap (with a 280-ms interval). Because the flow is split up at the trap, the maximal velocities in the looped main channel are reduced compared to the outlet. This reduction was used to determine the ratio of fluxes through the trap $\left(\mathrm{Q}_{\mathrm{TRAP}}\right)$ and through the main channel $\left(\mathrm{Q}_{\mathrm{MC}}\right)$ that are presented in (D) together with simulated values and the predictions based on the model proposed by Tan and Takeuchi. ${ }^{29}$ 


\section{Measurement of the fluidic resistance of a single cell trap}

We next performed particle image velocimetry (PIV) to determine the flow profile in the hydrodynamic trap. ${ }^{35,36,41}$ As it was expected, one fraction of the flow passed through the trap at a high speed, reducing the flow velocities of the beads in the looped main channel compared to the flow rates in the inlet (ESI movie $1 \dagger$ ). By quantifying this reduction for different trap designs (Fig. 2C), we calculated the ratio of the medium flux through the main channel and through the trap. This ratio increased from 0.9 (nominal 1) to 4.1 (nominal 4) (Fig. 2D).

For comparison, Fig. 2D also depicts the predicted and simulated ratio of the fluxes through the trap and the main channel. The predictions were calculated based on measurements done on confocal stacks (Fig. 1B). The measured data show a strong linear correlation $\left(\mathrm{R}^{2}=0.995\right)$ and agree well with the predictions put forward by Tan and Takeuki ${ }^{29}$ and with the values obtained from our simulation. The small deviation (about $20 \%$ for the $4-\mathrm{mm}$ design) of the model from the measured data is most probably due to errors in the dimensions used to calculate the model data. The fact that the measured flow ratios corresponded well with the predictions and the simulation (ESI Fig. $1 \dagger$ ) provides support for the previously published model to predict the flow ratio in the hydrodynamic trap. ${ }^{29}$ Thus, the fraction of the flow through a single cell trap can be modified by varying a single parameter such as the main channel length. Such control can be of importance when working with cells which can have, unlike beads, a rather wide size distribution.

\section{Quantification of the efficiency of single cell trapping}

To investigate how the trapping efficiency depended on the main channel length, non-adherent EG7 cells were loaded into single cell traps of the array and trapping yield, defined here as the number of successfully trapped cells per number of cells passing by next to a trap, was assessed (Fig. 3A, ESI movie 2 and $3 \dagger$ ). In order to record a sufficient number of trapping events, trapped cells were removed from the trap by reversing the flow which was sufficient to generate an unbiased population of cells (ESI movie 2 and $3 \dagger$ ). Indeed, we did not observe any difference in trapping efficiency during the cell loading and in the later time course of the experiment (data not shown).

A comparison of the three investigated trap designs shows that the trapping efficiency significantly increases from $63 \%( \pm 15 \%)$ for the 1 -mm design to $97.2 \%( \pm 2.6 \%)$ for the 4-mm design (Fig. 3B). Assuming that a trapping probability of $90 \%$ is sufficient to ensure efficient cell trapping, the ratio of the fluxes through the trap and the main channel must be at least $1: 2$, which is close to the previously postulated ratio of $1: 3 .^{29}$ Moreover, the trapping efficiency of our device correlates well with the ratio of the fluxes through the trap and the main channel $\left(\mathrm{R}^{2}=0.93\right)$. However, as Fig. 3B shows, this correlation is not exactly linear but reaches a plateau. That is, a further increase of the flux through the trap would not result in a higher trapping efficiency.

On the other hand, if the ratio of the fluxes through the trap and the main channel is too low, single cell trapping becomes more and more improbable, which may explain the low trapping yields associated with previously published single cell traps ${ }^{21,23,27}$ and indicates the potential of such a highly efficient single cell trap array for applications involving rare cells such as stem cells. For example, we were able to load an entire array of approximately 400 single cell traps by seeding less than 1000 hematopoietic stem cells (not shown).

\section{Long-term trapping of single cells}

Using live cell microscopy, we next studied the effect of the perfusion rate and of the main channel length on cell viability and on the long-term performance of the single cell trap (Fig. 4A and Fig. 4B). Due to the higher trapping efficiency of single cell traps with the 2- and 4-mm main channel lengths, only these designs were considered for these experiments.

Notably, in the 2-mm design we managed to stably trap single non-adherent cells, losing only ca. $22 \%( \pm 7.5 \%)$ at a flow rate of $100 \mathrm{nl} / \mathrm{min}$. Increasing or decreasing the flow rates significantly increased the number of lost cells (Fig. 4B and Fig. 4C) $(p<0.05)$. At higher flow rates, cells tended to squeeze through the trap more often, whereas at lower flow rates the perfusion in the device became less stable due to clogging resulting in an increased cell loss.

When perfusing the chip at a flow rate of $100 \mathrm{nl} / \mathrm{min}$, cell loss seemed to stabilize after around $3 \mathrm{~h}$ (Fig. 4B), which was not the case for the higher and lower perfusion rates. These observations indicate that cell losses can at least be partially explained by perturbations of the flow rates due to the handling of the microfluidic device. Once the perfusion stabilized, cell loss reached a stable level of $c a$. $75 \%$ constantly trapped cells. These
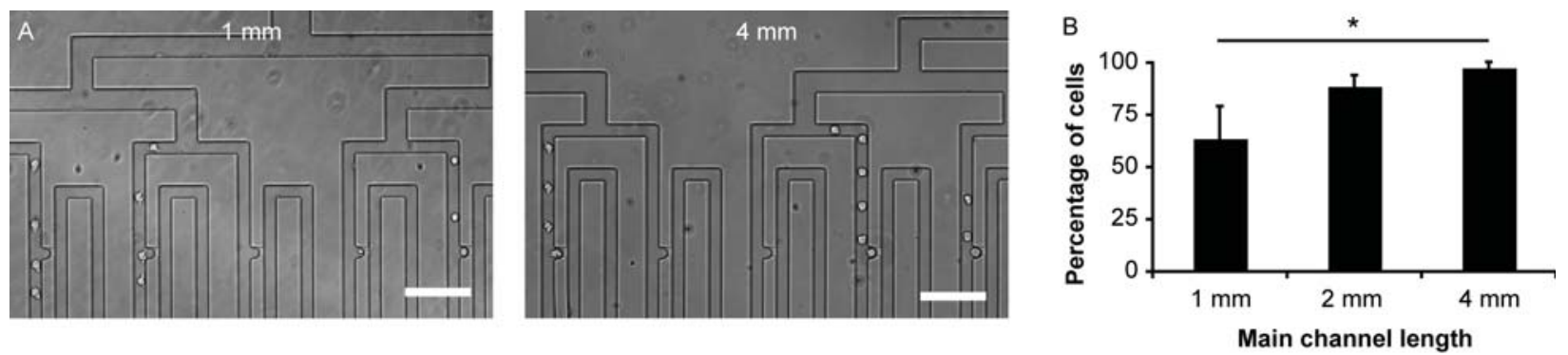

Fig. 3 Trapping efficiency in the microfluidic single cell trap. (A) Overlay of a series of images (taken at a 100-ms interval) of a 1-mm and a 4-mm trap design showing EG7 cells missing the trap (1-mm design) and being trapped (4-mm design). Scale bars are $100 \mu \mathrm{m}$. (B) Trapping yield for single traps with 1-mm, 2-mm and 4-mm long main channels demonstrating that the trapping efficiency increases proportionally with main channel length. Trapping efficiency was assessed as number of successfully trapped cells per number of cells arriving at a trap $(\mathrm{N}=100$ cells/chip in triplet). 

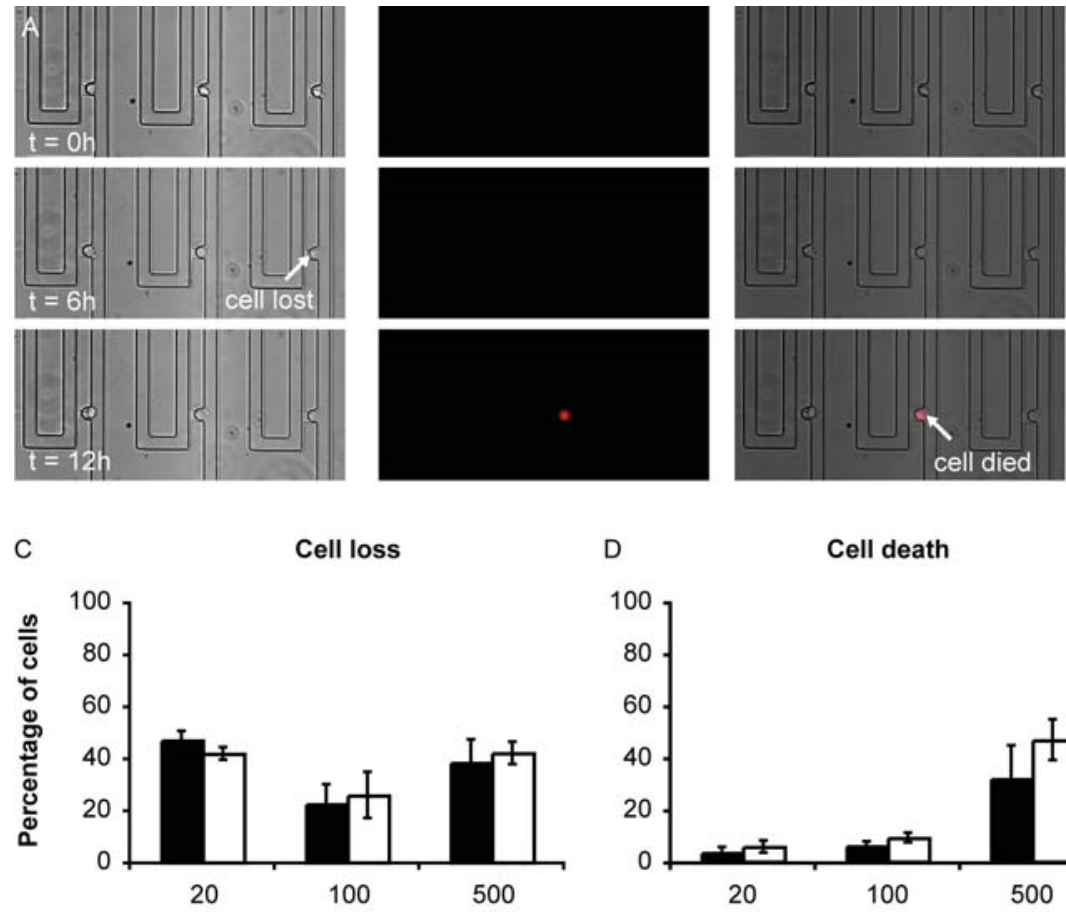

D

Cell death

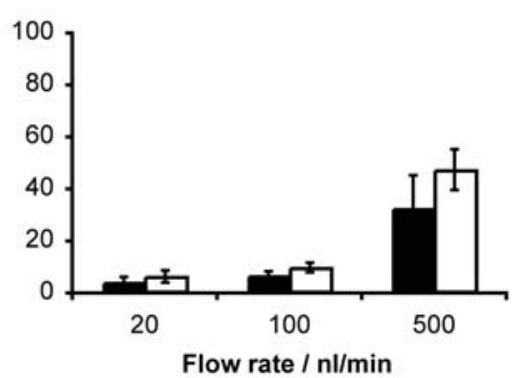

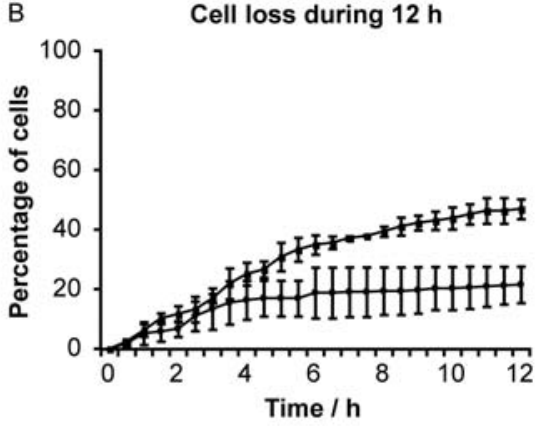

$\rightarrow 20 \mathrm{nl} / \mathrm{min} \rightarrow 100 \mathrm{nl} / \mathrm{min}$

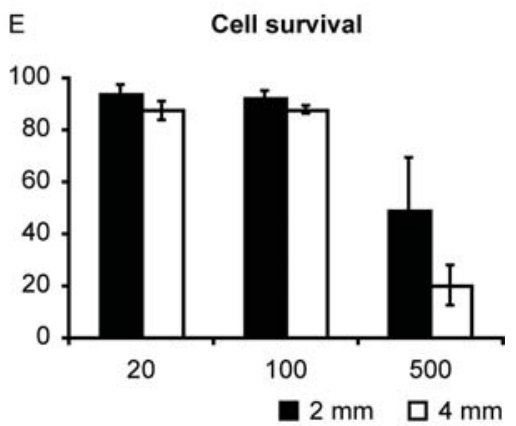

Fig. 4 Fates of non-adherent EG7 cells in a microfluidic single cell trap. (A) Series of typical images from a time-lapse experiment in a 2-mm device at a flow rate of $100 \mathrm{nl} / \mathrm{min}$ show a stably trapped cell and one that was lost after $6 \mathrm{~h}$ (bright-field image, left panel). Cell death was detected using propidium iodine (PI) added to the medium (fluorescence image in the middle and overlay in the right panel). (B) Example of the evolution of cell loss during $12 \mathrm{~h}$ in the 2-mm design at two different flow rates. (C) Cumulative cell loss, (D) total cell death and (E) cell survival of the stably trapped cells for the 2-mm and 4-mm design after $12 \mathrm{~h}$. Approximately 100 cells were analyzed for each condition (in triplicate).

numbers significantly exceed those reported earlier for nonadherent cells ${ }^{27,30}$ and nearly reach the levels of adherent cells. ${ }^{23}$

\section{Assessment of on-chip cell viability}

We then analyzed the viability of trapped single cells. For flow rates below $100 \mathrm{nl} / \mathrm{min}$ (equal to a flow velocity of $65 \mu \mathrm{m} / \mathrm{min}$ ), cell death in all analyzed cell traps was always less than $9 \%$, as it is the case for the $2-\mathrm{mm}$ design at $20 \mathrm{nl} / \mathrm{min}$ (Fig. 4D). This flow rate corresponds to a shear stress of about $2.4 \mathrm{mPa}$ and is comparable to shear stresses found in interstitial tissues. ${ }^{40}$ Accordingly, these findings are comparable to the outcome of static single cell culture in PEG microwell arrays (ESI Fig. $2 \dagger$ ) and to standard cell culture (data not shown), which has only been achieved in adherent single cell trapping ${ }^{23}$ but not for trapped non-adherent cells. ${ }^{27}$ However, an increase of the perfusion rate to $500 \mathrm{nl} / \mathrm{min}$ led to a significant increase of the number of PI-positive cells ( $\mathrm{p}<0.05)$, most probably evoked by higher shear stress of about $60 \mathrm{mPa}$, a value that is comparable to the shear stress found in the blood system. ${ }^{42}$

We did not detect any significant differences between the tested trap designs, but rather a slight trend towards better cell survival in the $2-\mathrm{mm}$ design compared to the $4-\mathrm{mm}$ design $(\mathrm{p}<0.1)$. The decreased cell viability may be due to the higher fluidic resistance and hence to the higher pressure difference across the trap in the 4-mm compared to the 2-mm design. More importantly, because the flow rates were identical throughout the entire array, the viability did not depend on the trap positions as was observed with other systems. ${ }^{27}$

\section{Automated separation of daughter cells upon division}

As an illustrative application for such a reliable single cell trap array, we investigated the division of single non-adherent cells within a series of 2-mm traps. We hypothetized that when trapping only a small number of single cells, we would be able to separate the daughter cells generated upon mother cell division. One daugher cell should remain in the trap and the other one
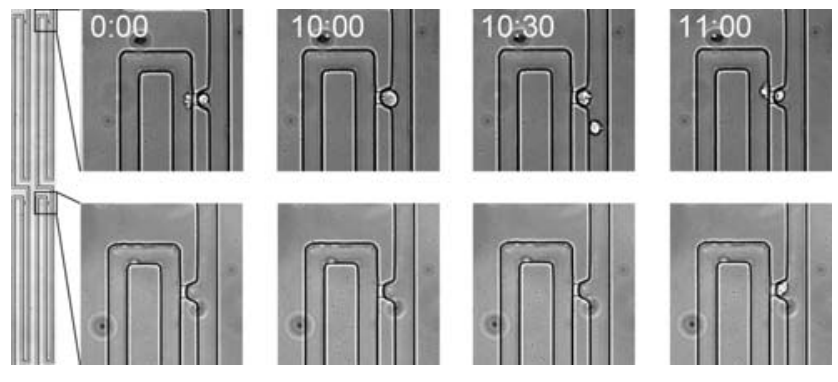

Fig. 5 Sections of time-lapse images (taken at a 30-min interval) showing the automated separation of daughter cells upon division. After completing the growth phase $(0: 00-10: 00)$ and division $(10: 30)$, one daughter cell remains in the first microfluidic single cell trap while the other is transported to the next free trap by perfusion (from the top) and captured therein $(11: 00)$. 
should be transported to the next empty trap by the medium flow. Indeed, as Fig. 5 shows, we could trap a few single cells and observe their growth over time. The daughter cells generated upon division of the trapped single cells could be readily trapped and thus the two daughter cells be separated. Although the proliferation of populations of trapped single cells and the image analysisbased tracking of dividing single cells were already described previously, ${ }^{8,23,43}$ the automated separation of daughter cells in a traceable manner was never reported ${ }^{23}$ and is currently only possible using labor-intensive (manual) micromanipulation. ${ }^{44}$

\section{Conclusions}

Here we systematically improved the efficiency and long-term performance of hydrodynamic single cell trapping. Our approach was based on a previously published model to predict the flow profile in single bead traps. ${ }^{29} \mathrm{We}$ first used computational models and flow profiling to verify and optimize the hydrodynamic conditions in our single cell trap. With these optimized trap architectures we demonstrated single cell trapping efficiencies of up to $97 \%$, which to the best of our knowledge was never reported for single cell trapping. Using time-lapse microscopy, we identified optimal perfusion rates that allowed both a high cell viability $(>90 \%)$ and a minimization of cell loss $(<23 \%)$.

This platform should be useful for the study of rare or heterogenous cell populations such as stem cells, tumor cells or hybridoma cells. The reliable trapping and re-trapping after cell divisions should for example open the door for novel types of investigations in mammalian cell biology, which depend on cell clonality and precise knowledge of lineage relationships and genealogy.

\section{Acknowledgements}

We thank Orestis Malaspinas for his support with the implementations of the lattice Boltzmann code and Samy Gobaa for help with data analyses. We also thank Marc Chambon (Biomolecular Screening Facility, EPFL) for excellent assistance with time-lapse experiments and Dusty Moore and Vincent Jaquet for their valuable help with flow characterization.

\section{Notes and references}

1 Y. Marcy, C. Ouverney, E. M. Bik, T. Lösekann, N. Ivanova, H. G. Martin, E. Szeto, D. Platt, P. Hugenholtz, D. A. Relman and S. R. Quake, Proc. Natl. Acad. Sci. U. S. A., 2007, 104, 11889-11894.

2 M. Lutolf, R. Doyonnas, K. Havenstrite, K. Koleckar and H. Blau, Integr. Biol., 2009, 1, 59-69.

3 T. C. Chao and A. Ros, J. R. Soc. Interface, 2008, 5(Suppl 2), S139150.

4 D. Di Carlo and L. P. Lee, Anal. Chem., 2006, 78, 7918-7925.

5 M. E. Lidstrom and D. R. Meldrum, Nat. Rev. Microbiol., 2003, 1, $158-164$.

6 A. Ogunniyi, C. Story, E. Papa, E. Guillen and J. Love, Nat. Protoc., 2009, 4, 767-782.

7 A. Khademhosseini, J. Yeh, S. Jon, G. Eng, K. Y. Suh, J. A. Burdick and R. Langer, Lab Chip, 2004, 4, 425-430.

8 S. Kobel, M. Limacher, S. Gobaa, T. Laroche and M. P. Lutolf, Langmuir: the ACS journal of surfaces and colloids, 2009.
9 M. R. Dusseiller, D. Schlaepfer, M. Koch, R. Kroschewski and M. Textor, Biomaterials, 2005, 26, 5917-5925.

10 H. Andersson and A. van den Berg, Curr. Opin. Biotechnol., 2004, 15, $44-49$.

11 R. M. Johann, Anal. Bioanal. Chem., 2006, 385, 408-412.

12 J. Voldman, Annu. Rev. Biomed. Eng., 2006, 8, 425-454.

13 J. S. Marcus, W. F. Anderson and S. R. Quake, Anal. Chem., 2006, 78, 3084-3089.

14 D. R. Albrecht, G. H. Underhill, T. B. Wassermann, R. L. Sah and S. N. Bhatia, Nat. Methods, 2006, 3, 369-375.

15 N. Mittal, A. Rosenthal and J. Voldman, Lab Chip, 2007, 7, 11461153.

16 M. He, J. S. Edgar, G. D. Jeffries, R. M. Lorenz, J. P. Shelby and D. T. Chiu, Anal. Chem., 2005, 77, 1539-1544.

17 T. Braschler, N. Demierre, E. Nascimento, T. Silva, A. G. Oliva and P. Renaud, Lab Chip, 2008, 8, 280-286.

18 F. Arai, A. Ichikawa, M. Ogawa, T. Fukuda, K. Horio and K. Itoigawa, Electrophoresis, 2001, 22, 283-288.

19 M. Ozkan, M. Wang, C. Ozkan, R. Flynn and S. Esener, Biomed. Microdevices, 2003, 5, 61-67.

20 U. Seger-Sauli, M. Panayiotou, S. Schnydrig, M. Jordan and P. Renaud, Electrophoresis, 2005, 26, 2239-2246.

21 A. R. Wheeler, W. R. Throndset, R. J. Whelan, A. M. Leach, R. N. Zare, Y. H. Liao, K. Farrell, I. D. Manger and A. Daridon, Anal. Chem., 2003, 75, 3581-3586.

22 A. Valero, J. N. Post, J. W. van Nieuwkasteele, P. M. Ter Braak, W. Kruijer and A. van den Berg, Lab Chip, 2008, 8, 62-67.

23 D. Di Carlo, L. Wu and L. P. Lee, Lab Chip, 2006, 6, 1445-1449.

24 J. Seo, C. Ionescu-Zanetti, J. Diamond, R. Lal and L. P. Lee, Appl. Phys. Lett., 2004, 84, 1973-1975.

25 M. Khine, A. Lau, C. Ionescu-Zanetti, J. Seo and L. P. Lee, Lab Chip, 2005, 5, 38-43.

26 P. J. Lee, P. J. Hung, R. Shaw, L. Jan and L. P. Lee, Appl. Phys. Lett., 2005, 86, 223902.

27 S. Faley, K. Seale, J. Hughey, D. K. Schaffer, S. VanCompernolle, B. McKinney, F. Baudenbacher, D. Unutmaz and J. P. Wikswo, Lab Chip, 2008, 8, 1700-1712.

28 D. Di Carlo, N. Aghdam and L. P. Lee, Anal. Chem., 2006, 78, 49254930.

29 W. Tan and S. Takeuchi, Proc. Natl. Acad. Sci. U. S. A., 2007, 104, 1146-1151.

30 D. Wlodkowic, S. Faley, M. Zagnoni, J. P. Wikswo and J. M. Cooper, Anal. Chem., 2009, 81, 5517-5523.

31 Y. N. Xia and G. M. Whitesides, Annu. Rev. Mater. Sci., 1998, 28, 153-184.

32 T. Braschler, L. Metref, R. Zvitov-Marabi, H. van Lintel, N. Demierre, J. Theytaz and P. Renaud, Lab Chip, 2007, 7, 420-422.

33 S. Chen and G. D. Doolen, Annu. Rev. Fluid Mech., 1998, 30, 329 364.

34 J. Latt, B. Chopard, O. Malaspinas, M. Deville and A. Michler, Phys. Rev. E: Stat., Nonlinear, Soft Matter Phys., 2008, 77, 056703.

35 J. G. Santiago, S. T. Wereley, C. D. Meinhart, D. Beebe and R. Adrian, Exp. Fluids, 1998, 25, 316-319.

36 L. Kim, M. D. Vahey, H. Y. Lee and J. Voldman, Lab Chip, 2006, 6, 394- 406.

37 D. J. Beebe, G. A. Mensing and G. M. Walker, Annu. Rev. Biomed. Eng., 2002, 4, 261-286.

38 B. Chopard and M. Droz, Cellular Automata Modeling of Physical Systems University Press Cambridge, 1998.

39 L. Landau and E. Lifshitz, Course of Theoretical Physics, Volume 6 (Fluid Mechanics), 2nd edn, Pergamon Press, Oxford, 1987.

40 J. M. Rutkowski and M. A. Swartz, Trends Cell Biol., 2007, 17, 44-50.

41 S. Devasenathipathy, J. G. Santiago, S. T. Wereley, C. D. Meinhart and K. Takehara, Exp. Fluids, 2003, 34, 504-514.

42 L. Chau, M. Doran and J. Cooper-White, Lab Chip, 2009, 9, 18971902.

43 R. J. Taylor, D. Falconnet, A. Niemistö, S. A. Ramsey, S. Prinz, I. Shmulevich, T. Galitski and C. L. Hansen, Proc. Natl. Acad. Sci. U. S. A., 2009, 106, 3758-3763.

44 H. Ema, H. Takano, K. Sudo and H. Nakauchi, J. Exp. Med., 2000, 192, 1281-1288. 